\title{
SELECTIVE DEPOSITION OF NANOFIBERS NET ON TEXTILE STRUCTURES
}

\section{DOI: 10.35530/TT.2021.34}

\section{Minguez, E.B. Belda, I. Montava, P.D. García, M.B. Aracil*, J.G. Payá}

DITEXPA, GIITEX, Universitat Politècnica de València, Spain

(E-mail: damingar@epsa.upv.es, evbobel@upv.es, imontava@txp.upv.es, pdiazga@txp.upv.es, maboar@txp.upv.es, jaigispa@txp.upv.es)

\begin{abstract}
Nanotechnology has evolved in the last years and nowadays there are many technologies related with the development of nanoparticles (NPs) or nanofibers (NFs). Due to the wide variety of polymers and diverse applications, filtration, medicine, cosmetic, etc., the study of those NFs is still of interest nowadays. In this work the NFs net created from electrospinning is used as a coating for fabrics. The aim of this work is to demonstrate how fibres are placed on the fabrics and if there is a tendency or they are located randomly. Two different fabrics were used a $100 \%$ cotton plain $115 \mathrm{~g} / \mathrm{m}^{2}$ and a $100 \%$ polyamide knitting fabric $60 \mathrm{~g} / \mathrm{m}^{2}$. A PVA solution (9\% $w / v)$ was used to create NFs which were placed on the fabrics. This solution was prepared by heating water at $80^{\circ} \mathrm{C}$ till complete solution of the polymer. Electrospinning was designed for a vertical collector with $15 \mathrm{~cm}$ distance from the needle. The flow rate was $0,5 \mathrm{~mL} / \mathrm{h}$ with 15 or $20 \mathrm{kV}$ for 15 minutes. Results evidenced the tendency of NFs net to be located on the fibres and consequently we could conclude the fabric is designing the position where the fibres would be placed. Furthermore, we could demonstrate that the presence of a fabric with reduced density implies deposition of NFs on both the fabric and the collector.
\end{abstract}

Keywords: electrospinning, located, nanofiber, substrate

\section{INTRODUCTION}

Nanotechnology has evolved in the last years and nowadays there are many technologies related with the development of nanoparticles (NPs) or with nanofibers (NFs). There is a wide field of application for NFs nets, they can be used for filtration $[1,2]$, biomedicine [3-5] or even for protection against COVID-19 [6-8].

NFs net can be made of different polymers such as polypropylene (PP) [9], polyamide (PA) [10], polyvinyl alcohol (PVA) [11], Polylactic acid (PLA) [12], etc.

Electrospinning is a technique based on the use of electrical forces to produce polymeric fibres of small diameter, comprised in a range that varies from micrometres (10$100 \mu \mathrm{m})$ to nanometres $\left(10 \times 10^{-3}-100 \times 10^{-3} \mu \mathrm{m}\right)$, and with a high specific area, in a relatively simple and inexpensive way [13].

The technique consists of the application of a high electrostatic field between a polymeric solution and a collecting surface. The positive pole is attached to an injection system, in this case the needle (metallic capillar) and the negative pole to a metallic plate or collector (connected to ground) which is generally between $5 \mathrm{~cm}$ and $30 \mathrm{~cm}$ away from the metallic capillar [13-14]. On the collector the nanometric fibres are deposited, forming a fabric (nonwoven) with a characteristic texture, density and color [14].

The liquid drop remains attached to the tip of the capillar thanks to the surface tension until the repulsion is greater and causes a force in the opposite direction to the contraction of the drop. As a consequence of this application, an electrified meniscus known as the Taylor 
Cone is formed, it is due to polarization and charge, causing a transverse and a normal force. As the cone accelerates the elongation process, it reaches a point where the tangential and normal components are equal, at this time the cone solidifies creating fibres and depositing them on the collector plate [13-14].

Some parameters affect electrospinning, they can be classified into three groups: dissolution parameters (polymer solution), process parameters and environment parameters. All of them influence the morphology and properties of the fibres [13-14].

\subsection{Solution properties}

- Concentration: it is one of the most determining parameters in the size and morphology of the fibres. Concentration affects both viscosity and surface tension. If the concentration is considerably high, apart from making it difficult for the solution to pass through the capillary, the diameter of the fibre may increase. On the other hand, if there is a low concentration of polymer, the solution will be so liquid that the drops will break before reaching the collecting plate due to the effect of surface tension [13-14].

- Molecular weight: The molecular weight of the polymer has a significant effect on rheology and electrical properties such as viscosity, surface tension, conductivity, and dielectric strength. It has been observed that low molecular weight solutions tend to form beads rather than fibres, and a high molecular weight one tends to form fibres with large diameters. The molecular weight also influences the number of polymer chain entanglements in a solution, therefore, the viscosity and entanglement of the chain play an important role in the electrospinning process [14-15].

- Viscosity: As it is also named in the concentration, the viscosity plays a very important role in electrospinning, when the viscosity is very low there will not be a continuous formation of fibre, the drops will break before reaching the collector. On the other hand, when the viscosity is relatively high, the polymer could present difficulties in its passage through the capillary [13-15].

- Surface tension: The surface tension will depend on the polymer and the solvent used. It has a relevant role in obtaining fibres without the presence of beads. The surface tension determines the upper and lower limits of the electric field, if all other variables are kept constant [14].

- Conductivity: The conductivity of the solution is determined by the type of polymer, the solvent used, and the availability of ionizable salts [14]. Solutions with high conductivity will have greater capacity to carry charges than those with low conductivity. The addition of salts to the solution increases the conductivity and therefore the electrical force for stretching the jet, which means obtaining fibres with a smaller diameter [13]. The lack or low electrical conductivity of the solution causes the elongation of the jet by the electrical force to be insufficient, and therefore, prevents the production of uniform fibres.

- Dielectric effect of the solvent: The solvent performs two jobs within the electrospinning process; first, it dissolves the polymer molecules to form the electrically charged jet, and second, it carries the dissolved polymer molecules to the collector [13].

Because of this, generally a solution with good dielectric properties reduces the bead formation and also the diameter of the resulting fibres.

\subsection{Process parameters}

They are the parameters that can be modified during the process. These are parameters that can be easily manipulated directly from the displays of the machine that we use.

- Voltage (applied voltage): Voltage is one of the most fundamental parameters, since only after reaching the threshold voltage does the formation of fibres occur [14]. It has been shown that when applying higher stress values, a greater stretching of the solution is allowed since the electrostatic repulsive force (Culomb force) increases, causing a reduction in the 
diameter of the fibres. Also increasing the voltage favours the rapid evaporation of the solvent. On the other hand, increasing the voltage is directly related with increasing the probability of obtaining fibres with defects (beads) [13-14].

- Flow: The flow or outlet flow is also an important parameter of the process. A lower flow rate would be desirable as it gives the solvent more time to evaporate, thus preventing the formation of defects in the fibre. On the other hand, when the outflow increases there is an increase in the diameter of the fibres and in the size of the defects. The minimum flow rate to achieve a stable Taylor cone is the one that should be maintained to avoid fibre defects and allow the solvent to evaporate [13-14].

- Needle-collector distance: A minimum distance is required between the needle and the collector; this is the minimum distance that must be given to the fibre to allow the solvent to evaporate. On the other hand, over very long distances, the fibres can break under their own weight or bead formation.

\subsection{Room parameters}

- Temperature: Temperature can decrease the evaporation time of the solvent. In addition, there is a direct relationship between temperature and viscosity, the higher the temperature, the lower the viscosity. The decrease in viscosity, as it was explained previously, can cause a decrease in the diameter of the fibre [13-14].

- Humidity: High humidity in the environment can cause small pores on the surface of the fibres due to the condensed water deposited on them as they go from the needle to the collector, having an influence on the morphology of the fibres, especially when working with volatile solvents [13]. On the other hand, a low humidity rate can cause the solvent to evaporate very quickly, causing that in some cases the process must be carried out in the shortest possible time in order to prevent the solution is dried at the tip of the needle obstructing it [14].

Due to the wide variety of polymers and diverse applications the study of those NFs is still of interest nowadays. In this work the NFs is used as a coating of fabrics. The aim of this work is to demonstrate how PVA NFs are placed on the fabrics surface and if there is a tendency created by the geometry of the collector surface, or they are located randomly despite the surface roughness on the collector. Table 1 summarizes the most important parameters with influence on NFs.

Table 1. Summary of parameters influence on NFs.

\begin{tabular}{|c|c|c|}
\hline PARAMETER & Tendency & Behaviour \\
\hline \multicolumn{3}{|c|}{ Solution parameters } \\
\hline \multirow{2}{*}{ Concentration } & Increase & Difficult for the solution to pass through the capillary \\
\hline & Decrease & Drops before reaching the collector \\
\hline \multirow{2}{*}{ Molecular weight } & Increase & Difficult for the solution to pass through the capillary \\
\hline & Decrease & Drops before reaching the collector \\
\hline \multirow{2}{*}{ Viscosity } & Increase & Difficult for the solution to pass through the capillary \\
\hline & Decrease & Drops before reaching the collector \\
\hline \multirow{2}{*}{ Surface tension } & Increase & No beads in NFs \\
\hline & Decrease & Beads presence in NFs \\
\hline \multirow[b]{2}{*}{ Conductivity } & Increase & Reduction in fibres diameter \\
\hline & Decrease & $\begin{array}{l}\text { Electrostatic forces derive into jet enlargement and it } \\
\text { is not possible to obtain NFs }\end{array}$ \\
\hline \multicolumn{3}{|r|}{ Process parameters } \\
\hline \multirow{2}{*}{ Voltage } & Increase & Thick fibres, jet distosion, beds presence \\
\hline & Decrease & Solution does not reach the collector \\
\hline \multirow{3}{*}{ Flow rate } & Increase & Thicker fibres, higher size for beds. \\
\hline & Decrease & $\begin{array}{l}\text { Solvent evaporation takes longer, homogeneous } \\
\text { fibres. }\end{array}$ \\
\hline & Increase & Broken fibres due to its weight. \\
\hline
\end{tabular}




\begin{tabular}{|c|l|l|}
\hline \multirow{2}{*}{$\begin{array}{c}\text { Needle-collector } \\
\text { distance }\end{array}$} & $\begin{array}{l}\text { Higher enlargement of the solution. } \\
\text { Thinner fibres }\end{array}$ \\
\cline { 2 - 3 } & Decrease & $\begin{array}{l}\text { Not enough time for solvent evaporation, wet fibres } \\
\text { reach the collector. }\end{array}$ \\
\hline \multirow{2}{*}{ Temperature } & \multicolumn{2}{|c|}{ Environment parameters } \\
\cline { 2 - 3 } & Increase & $\begin{array}{l}\text { Solvent evaporates too quickly and dry polymer } \\
\text { appears in the needle. }\end{array}$ \\
\cline { 2 - 3 } Humidity & Decrease & $\begin{array}{l}\text { Solvent does not evaporate. Difficult for the solution } \\
\text { to pass through the capillary }\end{array}$ \\
\cline { 2 - 3 } & Increase & Solvent does not evaporate properly. Porous NFs \\
\hline Decrease & $\begin{array}{l}\text { Fast evaporation of solvent. Dry polymer appears in } \\
\text { the needle. }\end{array}$ \\
\hline
\end{tabular}

\section{MATERIALS AND METHODS}

\subsection{Materials}

A Nanospider system supplied by Bioinicia was used to produce the nanofibers. Nanofibers were made of Polyvinyl alcohol (PVA) Mw 61000 g/mol, supplied by SigmaAldrich. Solutions were prepared with distilled water.

The collector of the Nanospider system was covered by different fabrics. One of the fabrics was a plain fabric $100 \%$ cotton, $115 \mathrm{~g} / \mathrm{m}^{2}$. The other one was a $100 \%$ polyamide knitting fabric $60 \mathrm{~g} / \mathrm{m}^{2}$.

\subsection{Methods}

Nanofibers were electro spun form polymer solution containing $9 \% \mathrm{w} / \mathrm{v}$ which was prepared with PVA an distilled water at $80^{\circ} \mathrm{C}$ until complete solution. The electrospinning process was conducted with the same Nozzle-collector distance $(15 \mathrm{~cm})$ The collector was placed vertically. Identical flow rate $(0.5 \mathrm{ml} / \mathrm{h})$ was used but different Voltage (12, 15 or 20 $\mathrm{kV}$ ) for 15 minutes.

Scanning Electron Microscopy (SEM) was used to observe the fabrics surface and the NFs formation. FIB microscope from Zeiss was used as a Scanning Electron Microscopy (SEM) to analyse the fabrics' surface at $1.5 \mathrm{kV}$ and a suitable magnification. Samples were previously sputtered with a gold/platinum coating.

\section{RESULTS AND DISCUSSION}

As it has been explained in the introduction section the nanofibers are placed on a collector. In this study we covered the collector system with two different fabrics, a weave with a plain rapport, and a Knitting one with low density. The elctrospun fibres were placed on different fabrics a plain wave or a knitting one. Initially the voltage should be adapted in order to avoid the presence of beads on the NFs. Figure 1a shows the presence of polymer on the cotton fibres without NFs formation when the voltage is $12 \mathrm{kV}$. Polyamide fabric (60 $\left.\mathrm{g} / \mathrm{m}^{2}\right)$ worked perfectly with $15 \mathrm{kV}$ but cotton due to its higher density $\left(115 \mathrm{~g} / \mathrm{m}^{2}\right)$ interfered on the voltage at the collector and $15 \mathrm{kV}$ produced NFs but plenty of beads (figure $1 \mathrm{~b}$ ), and consequently it should be increased up to $20 \mathrm{kV}$ (figure 1, c).

Figure 2 shows the microscopy images where it can be appreciated the coating of PVA nanofibers regularly placed on the surface of cotton fibres and with no beds. Results evidenced that the nanofibers were placed on the fabric acting as a nanospider net coating the fabric, which was also demonstrated by some authors [11]. This web of NFs is placed on the collector or on the fabric when the fabric is covering the collector. 


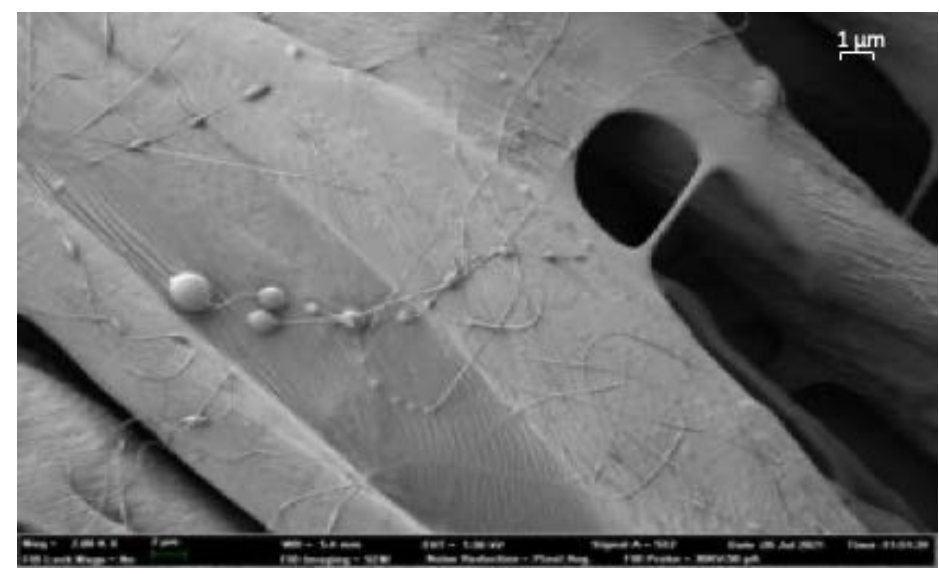

$\boldsymbol{a}$

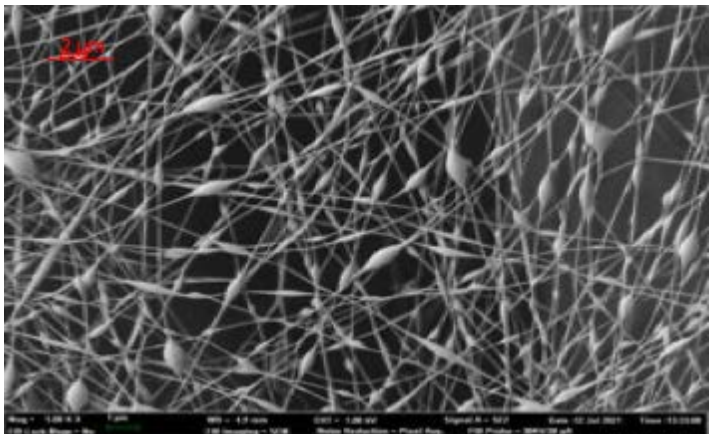

b

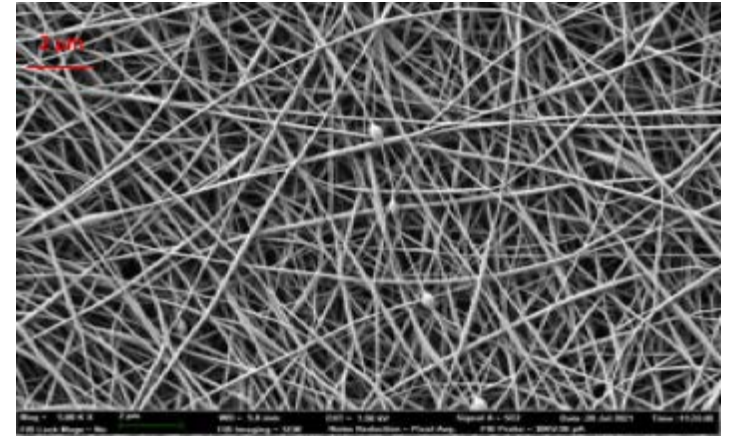

C

Figure 1. PVA nanofibers coating cotton fibres at different voltage: a - $12 \mathrm{kV}$; b - $15 \mathrm{kV}$; c - $20 \mathrm{kV}$

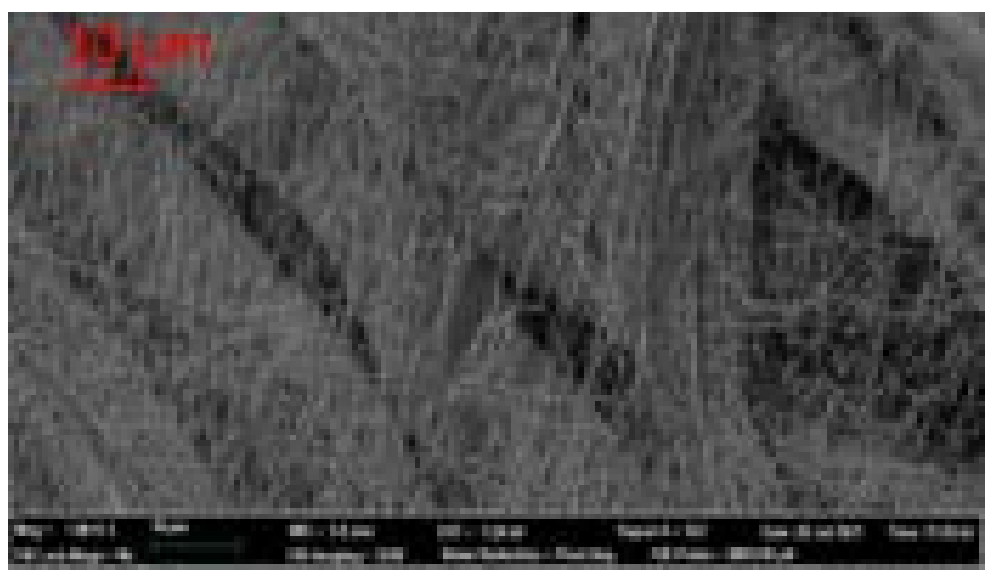

Figure 2. PVA nanofibers coating cotton fibres

When we compare the behaviour of the coating regarding the two fabrics, we can observe (figure 3) the nanofibers are placed basically on the fabric fibres, and we can clearly observe there are no nanofibers on the space between yarns. There was an evident difference in density between fabrics $115 \mathrm{~g} / \mathrm{m}^{2}$ for the weave one and $60 \mathrm{~g} / \mathrm{m}^{2}$ for the knitted one. This implies the space between yarns (holes) is considerably bigger for the knitted fabric. 


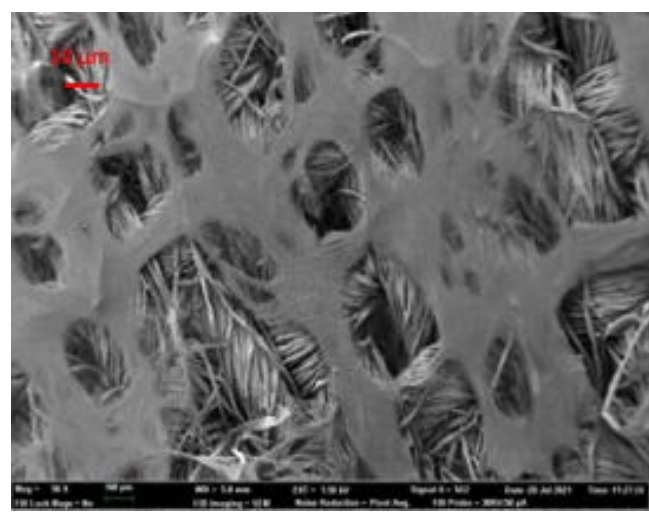

a

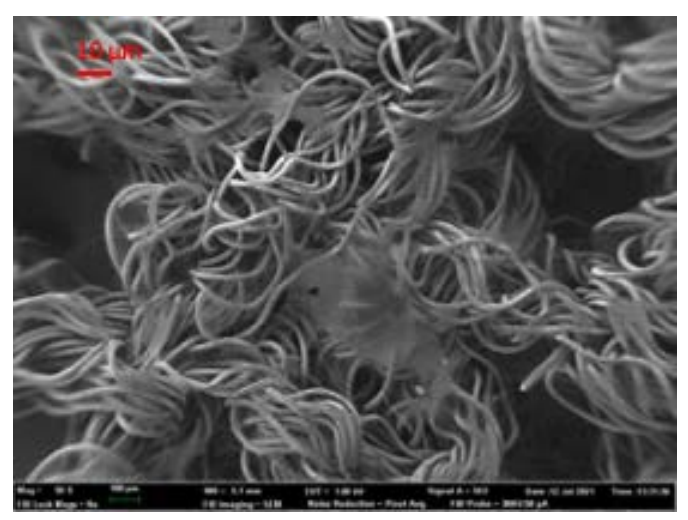

b

Figure 3. PVA nanofibers coating fabrics: a - plain fabric; b - knitted fabric

Nanofibers width is measured and results reveal there is a similar value obtained for nanofibers deposited on both fabrics, $128 \mathrm{~nm}$ for the weaving and $114 \mathrm{~nm}$ for the knitting. As the NFs are deposited on the fibres, it is clearly observed there is a higher presence of the nonwoven on the weave than on the knitted fabric due to the higher number of fibres. This is not due to the fabric technology used, weaving or knitting structure it is due to the presence of yarns. Weaving or knitting will only influence on the paths created by the nanofibers.

Figure 4 shows the appearance of the collector once the knitting fabric was removed. It is clearly appreciated a spotted surface. The spots observed can be attributed to the nanofibers which were not placed on the knitting fabric as there was a small holes or spaces between yarns.

Figure 4. Spotted collector once the knitted fabric is removed from collector

It has been demonstrated there is a clear tendency of NFs to be deposited on the fibres from the fabrics. Figure 3 evidences the grid formed by the NFs net on the plain fabric created. Furthermore, the results showed it is possible to create a micronets on the collector when the fabric shows reduced density of yarns, as the NFs pass through the fabric pores.

\section{CONCLUSION}

The observation of fabric surface at low enlargement, once nanofibers coating was placed on the fabric's surface evidenced the effect that the substrate has on the deposition of nanofibers onto the fabric. Despite being the collector the one which attracts the polymer we have demonstrated that there is a preference for nanofibers to be placed on the yarns of the fabrics, and not only on the collector. Thus, when the density of yarns is not too high the nanofibers are placed both on the collector and on the fabric fibres.

This behaviour allows to conclude that a suitable design with a specific raport can deal 
into a selective coating of nanofibers which can be used for different applications such as selective filtration, electric conductivity circuits, etc., or even to create specific NFs nets with concrete shapes throughout the pores of a fabric.

\section{ACKNOWLEDGMENT}

The authors would like to express appreciation for the support of the sponsor. The research has been funded by the INSTITUTO VALENCIANO DE COMPETITIVIDAD (IVACE), with the nominative line in favour of the Universitat Politècnica de València, approved by the Budget Law of the Generalitat Valenciana on the year 2021.

\section{REFERENCES}

[1] Wang, X., Xiang, H., Song, C., Zhu, D., Sui, J., Liu, Q., Long, Y., Highly efficient transparent air filter prepared by collecting-electrode-free bipolar electrospinning apparatus, In: Journal of hazardous materials, 2020, 385, 121535.

[2] Liu, C., Hsu, P. C., Lee, H. W., Ye, M., Zheng, G., Liu, N., Cui, Y., Transparent air filter for highefficiency PM 2.5 capture, In: Nature communications, 2015, 6, 1, 1-9

[3] Whang, C., Wang, X., Zhang, E., Yang, L., Yuan, H., Tu, W., Zhang, Y., An epigenetic bioactive composite scaffold with well-aligned nanofibers for functional tendon tissue engineering. In: Acta biomaterialia, 2018, 66,141-156

[4] Ren, G., Xu, X., Liu, Q., Cheng, J., Yuan, X., Wu, L., Wan, Y., Electrospun poly (vinyl alcohol)/glucose oxidase biocomposite membranes for biosensor applications, In: Reactive and Functional Polymers, 2006, 66, 12, 1559-1564

[5] Si, Y., Zhang, Z., Wu, W., Fu, Q., Huang, K., Nitin, N., Sun, G., Daylight-driven rechargeable antibacterial and antiviral nanofibrous membranes for bioprotective applications, In: Science advances, 2018, 4, 3, eaar5931

[6] Khanzada, H., Salam, A., Qadir, M. B., Phan, D. N., Hassan, T., Munir, M. U., Kim, I. S., Fabrication of Promising Antimicrobial Aloe Vera/PVA Electrospun Nanofibers for Protective Clothing, In: Materials, 2020, 13, 17, 3884

[7] Zhang, L., Li, L., Wang, L., Nie, J., \& Ma, G., Multilayer electrospun nanofibrous membranes with antibacterial property for air filtration, In: Applied Surface Science, 2020, 145962

[8] Chowdhury, M. A., Shuvho, M. B. A., Shahid, M. A., Haque, A. M., Kashem, M. A., Lam, S. S., Mofijur, M., Prospect of biobased antiviral face mask to limit the coronavirus outbreak, In: Environmental Research, 2020, 192, 110294

[9] Cho, D., Zhou, H., Cho, Y., Audus, D., Joo, Y.L., Structural properties and superhydrophobicity of electrospun polypropylene fibers from solution and melt, In: Polymer, 2010, 51, 25, 6005-6012

[10] Heikkilä, P., Harlin, A., Parameter study of electrospinning of polyamide-6, In: European Polymer Journal, 2008, 44, 10, 3067-3079

[11] Koski, A., Yim, K., Shivkumar, S., Effect of molecular weight on fibrous PVA produced by electrospinning, In: Materials Letters, 2004, 58, 3-4, 493-497

[12] Chen, Y., Lin, J., Fei, Y., Wang, H., Gao, W., Preparation and characterization of electrospinning PLA/curcumin composite membranes, In: Fibers and Polymers, 2010, 11, 8, 11281131

[13] Diaz, R.E., Micro/Nanofibras coaxiales de PLA/PEG para el encapsulamiento de microorganismos de interés biomédico y alimentario, In: Enginyeria Química, Escola Tècnica Superior d'Enginyeria Industrial de Barcelona, 2015

[14] Gandía, A.S., Estudio del proceso de electrohilatura del DL-PLG para optimizar su biodegradación en aplicaciones médicas, In: Ingeniería química, Universitat Politècnica de València, 2016

[15] Bhardwaj, N., Kundu, S.C., Electrospinning: A fascinating fiber fabrication technique, 2010, 28, 325-347. 